\title{
RATIONAL INTERPOLATION OF FUNCTIONS ON THE UNIT CIRCLE
}

\author{
Xin Li \\ Dedicated to Dick Askey on the occasion of his sixty-fifth birthday
}

\begin{abstract}
We establish various results on the convergence of a sequence of rational functions that interpolate a function on the unit circle. In particular, we extend Walsh's equi-convergence theorem and the Walsh-Sharma theorem on $L_{2}$ convergence (a special case of a theorem of Lozinski) for the interpolating rational functions.
\end{abstract}

\section{Introduction}

Let $\mathbf{T}$ be the unit circle in the complex plane $\mathbf{C}$, and let $\mathbf{D}$ and $\overline{\mathbf{D}}$ denote the open and closed unit disks, respectively. Also, let $z_{n, k}=e^{i \theta_{n, k}}, 0 \leq \theta_{n, 0}<\theta_{n, 1}<\cdots<$ $\theta_{n, n}<2 \pi$, and for a function $f$ defined on $\mathbf{T}$, let $L_{n}(f ; \cdot) \in \mathcal{P}_{n}$, the set of polynomials of degree at most $n$, such that $L_{n}\left(f ; z_{n, k}\right)=f\left(z_{n, k}\right)$ for $k=0,1, \ldots, n$. As early as in 1884, Méray showed that if $\left\{z_{n, k}: k=0,1, \ldots, n\right\}$ are the $n+1$ st roots of unity, i.e., $\theta_{n, k}=2 k \pi /(n+1), k=0,1, \ldots, n$, then the polynomials $L_{n}(f ; \cdot)$ do not necessarily converge to $f$. Indeed, Méray took the function $f_{M}(z)=1 / z$. So, $L_{n}\left(f_{M} ; z\right)=z^{n}$, $n=1,2,3, \ldots$. These polynomials do not approach the function $f_{M}(z)$ for all $z \in \mathbf{D}$, as $n \rightarrow \infty$, but approach the limit zero. Méray's example was examined later by Walsh [24] who pointed out that the singularity of $f_{M}(z)=1 / z$ at $z=0$ caused the sequence $\left\{L_{n}\left(f_{M} ; \cdot\right)\right\}$ to fail to approach $f_{M}(z)$ for $z \in \mathbf{D}$. Walsh also furnished the following lovely companion to Méray's example.

Walsh's Theorem (1932, [24, Theorem 1]). Let $f$ be continuous (or more generally Riemann integrable) on $\mathbf{T}$, and let $\left\{z_{n, k}: k=0,1, \ldots, n\right\}$ be the $n+1$ st roots of unity. Then the sequence $\left\{L_{n}(f ; \cdot)\right\}$ converges to the limit

$$
f_{\mathbf{D}}(z)=\frac{1}{2 \pi i} \int_{\mathbf{T}} \frac{f(t) d t}{t-z}
$$

for $z \in \mathbf{D}$, uniformly on any compact subset of $\mathbf{D}$.

It is worthwhile to recall some steps in the historical development prior to Walsh's Theorem. Runge $(1904,[19])$ proved that if $\left\{z_{n, k}: k=0,1, \ldots, n\right\}$ are the $n+1$ st roots of unity and if $f$ is analytic on $\overline{\mathbf{D}}$, then the sequence $\left\{L_{n}(f ; z)\right\}$ converges to $f(z)$ on $\overline{\mathbf{D}}$. After that, Fejér $(1918,[9])$ showed that $\left\{L_{n}(f ; z)\right\}$ converges to $f(z)$ for $z \in \mathbf{D}$ if $f(z)$ is assumed only to be continuous on $\overline{\mathbf{D}}$ and analytic in $\mathbf{D}$. See also [11].

Received April 2, 1998, revised August 24, 1998.

1991 Mathematics Subject Classification: 41A20, 41A05, 42A15.

Key words and phrases: interpolation, approximation by rational functions, orthogonal rational functions. 
There were further developments after Walsh's Theorem. When the points of interpolation are the roots of unity, Lozinski $(1940,[15])$ proved, under Fejér's assumption, the $L_{p}(p>0)$ convergence of $\left\{L_{n}(f ; \cdot)\right\}$ on the unit circle, i.e.,

$$
\lim _{n \rightarrow \infty} \int_{\mathbf{T}}\left|f(z)-L_{n}(f ; z)\right|^{p}|d z|=0, \quad p>0 .
$$

This result, when $p=2$, was rediscovered later by Walsh and Sharma (1964, [26]). Some more recent results on interpolation at the roots of unity can be found in the work of Saff and Walsh [21], Cavaretta, et al. [6, 7], and Sharma and Vertesi [22].

Walsh gave an example to show that when the points of interpolation $\left\{z_{n, k}\right\}$ are not the roots of unity, $\left\{L_{n}(f ; z)\right\}$ may fail to converge in $\mathbf{D}$ even though $\left\{z_{n, k}\right\}$ are sufficiently dense on $\mathbf{T}$.

Walsh's Example ([25, pp. 293-294]). Let $z_{n, k}$ be the roots of

$$
\left(\frac{1-\alpha z}{\alpha-z}\right)^{n+1}=1, \quad \alpha>1
$$

Let $f_{W}(z)=1 /(\zeta+\beta), 0<\beta<1$, where $\zeta=(1-\alpha z) /(\alpha-z)$. Then

$$
L_{n}\left(f_{W} ; z\right)=\frac{1}{\zeta+\beta}+\frac{(\alpha+\beta)^{n}\left(\zeta^{n+1}-1\right)}{\left[(-1)^{n}+\beta^{n+1}\right](\zeta+\beta)(\zeta-\alpha)^{n}},
$$

which converges for $z$ in only a part of the unit disk $\overline{\mathbf{D}}$.

Walsh's example shows that the distribution of the points of interpolation $\left\{z_{n, k}\right\}$ on $\mathbf{T}$ should be taken into account when we study the convergence of interpolating polynomials $\left\{L_{n}(f ; \cdot)\right\}$. Closely related to this observation, the following result is now well known (cf. [25, Chapter 7]).

The Uniform Distribution Theorem. A necessary and sufficient condition for

$$
\lim _{n \rightarrow \infty} L_{n}(f ; z)=f(z),
$$

uniformly for $z \in \overline{\mathbf{D}}$, for every $f$ analytic on $\overline{\mathbf{D}}$, is that the sequence of points $\left\{z_{n, k}: k=0,1, \ldots, n\right\}$ is uniformly distributed on $\mathbf{T}$, i.e.,

$$
\lim _{n \rightarrow \infty}\left|\prod_{k=0}^{n}\left(z-z_{n, k}\right)\right|^{1 / n}=|z|, \quad|z|>1 .
$$

Now, let us take a closer look at the points of interpolation $\left\{z_{n, k}\right\}$ in Walsh's example, which satisfy, for $|z|>1$,

$$
\lim _{n \rightarrow \infty}\left|\prod_{k=0}^{n}\left(z-z_{n, k}\right)\right|^{1 / n}=\lim _{n \rightarrow \infty}\left|\frac{(1-\alpha z)^{n+1}-(\alpha-z)^{n+1}}{(-\alpha)^{n+1}-(-1)^{n+1}}\right|^{1 / n}=\left|z-\frac{1}{\alpha}\right| .
$$

They are not uniformly distributed unless $\alpha=\infty$. How do we recover a function $f$ analytic on $\overline{\mathbf{D}}$ if we are given the values of $f$ at the points $\left\{z_{n, k}\right\}$ as in Walsh's example? According to the Uniform Distribution Theorem, the interpolating polynomials do not necessarily converge to $f$ on $\overline{\mathbf{D}}$; we must use interpolating functions that are more general than polynomials. Indeed, in Walsh's example, we just need to use rational functions of the form

$$
\frac{p_{n}(z)}{(\alpha-z)^{n+1}}, \quad p_{n} \in \mathcal{P}_{n}
$$


that interpolate $f$ at $\left\{z_{n, k}: k=0,1, \ldots, n\right\}$. Then, by a simple transformation $z=(1-\alpha \zeta) /(\alpha-\zeta)$, Runge's result mentioned earlier would imply that this sequence of interpolating rational functions converges to $f$ on $\overline{\mathbf{D}}$. It is the purpose of this paper to explore this direction further and hence extend Walsh's Theorem to interpolation on the unit circle $\mathbf{T}$ by rational functions.

\section{More Notation}

Let $\alpha_{n}, n=0,1, \ldots$, be given (not necessarily distinct) points in $\mathbf{D}$, and let

$$
w_{n}(z):=\prod_{k=0}^{n}\left(z-\alpha_{k}\right) \text { and } w_{n}^{*}(z):=\prod_{k=0}^{n}\left(1-\overline{\alpha_{k}} z\right) .
$$

We now define the space of rational functions for each $n$. Let

$$
\mathcal{R}_{n}:=\left\{\frac{p(z)}{w_{n}^{*}(z)}: p \in \mathcal{P}_{n}\right\}
$$

and let

$$
\mathcal{R}_{n}^{*}:=\left\{\overline{r(1 / \bar{z})}: r \in \mathcal{R}_{n}\right\}
$$

Then we see that, when we restrict $z$ to $\mathbf{T}$, we have $\mathcal{R}_{n}^{*}=\left\{\bar{r}: r \in \mathcal{R}_{n}\right\}$. In general, an element in $\mathcal{R}_{n}^{*}$ has the form

$$
\frac{z p(z)}{w_{n}(z)} \quad \text { for some } p \in \mathcal{P}_{n}
$$

Define

$$
\mathcal{R}=\bigcup_{n=0}^{\infty} \mathcal{R}_{n} \quad \text { and } \quad \mathcal{R}^{*}=\bigcup_{n=0}^{\infty} \mathcal{R}_{n}^{*} .
$$

We will write $B_{n}(z)=w_{n}(z) / w_{n}^{*}(z)$. From now on, we will let the points of interpolation $\left\{z_{n, k}: k=0,1, \ldots, n\right\}$ be given as the roots of

$$
w_{n}(z)=w_{n}^{*}(z) \quad \text { or, equivalently, } \quad B_{n}(z)=1 .
$$

It is easy to see that $z_{n, k} \in \mathbf{T}$ for all $k=0,1, \ldots, n$ and $n=1,2, \ldots$ Moreover, the $z_{n, k} \mathrm{~s}$ are distinct as implied by formula (9) to be given later. For a function $f$ defined on $\mathbf{T}$, let $R_{n}(f ; \cdot)$ denote the unique rational function from $\mathcal{R}_{n}$ that interpolates $f$ at $\left\{z_{n, k}: k=0,1, \ldots, n\right\}$. Then, it is easy to verify that

$$
R_{n}(f ; z)=\sum_{k=0}^{n} \frac{f\left(z_{n, k}\right)\left(B_{n}(z)-1\right)}{B_{n}^{\prime}\left(z_{n, k}\right)\left(z-z_{n, k}\right)} .
$$

We will use $C(\mathbf{T})$ to denote the space of functions continuous on $\mathbf{T}$ equipped with the sup-norm on $\mathbf{T}$. Let $A(\mathbf{D})$ denote the disk algebra, that is, the set of functions continuous on $\overline{\mathbf{D}}$ and analytic in $\mathbf{D}$.

Finally, let

$$
\sigma_{n}:=\sum_{k=0}^{n}\left(1-\left|\alpha_{k}\right|\right) \quad \text { and } \quad \delta_{n}:=\min _{0 \leq k \leq n}\left(1-\left|\alpha_{k}\right|\right) .
$$




\section{Main results}

We will establish various convergence results of the sequence $\left\{R_{n}(f ; \cdot)\right\}$ according to different assumptions on $f$. First, we give a simple and natural extension of Walsh's Theorem to rational interpolation.

Theorem 1. Assume that $\lim _{n \rightarrow \infty} \sigma_{n}=\infty$. If $f \in C(\mathbf{T})$, then

$$
\lim _{n \rightarrow \infty} R_{n}(f ; z)=f_{\mathbf{D}}(z)
$$

for $z \in \mathbf{D}$, and the convergence is uniform on any compact subset of $\mathbf{D}$.

We remark that the requirement $\lim _{n \rightarrow \infty} \sigma_{n}=\infty$ is equivalent to saying $\lim _{n \rightarrow \infty}$ $B_{n}(z)=0$ for $z \in \mathbf{D}$ (see, e.g., $[25, \S 10.1]$ ). It is also a necessary and sufficient condition for the denseness of certain rational functions in $C(\mathbf{T})$ (cf. $[1,12])$; see Lemma 3 for a more precise statement.

Our proof of Theorem 1 depends on the following result of weak-star convergence of a sequence of discrete measures supported at the points of interpolation. Let $d \delta_{z}$ denote the unit measure supported at the single point $z$. For $n=0,1,2, \ldots$, define

$$
d \nu_{n}=\sum_{k=0}^{n} \frac{d \delta_{z_{n, k}}}{\left|B_{n}^{\prime}\left(z_{n, k}\right)\right|}
$$

Theorem 2. If $\lim _{n \rightarrow \infty} \sigma_{n}=\infty$, then $\left\{d \nu_{n}\right\}$ converges in the weak-star topology to the uniform measure on $\mathbf{T}, d \theta /(2 \pi)$. More precisely,

$$
\lim _{n \rightarrow \infty} \sum_{k=0}^{n} \frac{f\left(z_{n, k}\right)}{\left|B_{n}^{\prime}\left(z_{n, k}\right)\right|}=\frac{1}{2 \pi} \int_{0}^{2 \pi} f\left(e^{i \theta}\right) d \theta
$$

for every $f \in C(\mathbf{T})$.

Note that when $\alpha_{n}=0$ for all $n=0,1,2, \ldots$, we have $B_{n}(z)=z^{n+1}$ and $z_{n, k}$ are the $n+1$ st roots of unity, and $\left|B_{n}^{\prime}(z)\right|=n+1$ for $z \in \mathbf{T}$. So, equation (2) becomes the well-known equality

$$
\lim _{n \rightarrow \infty} \sum_{k=0}^{n} \frac{f\left(e^{2 i \pi /(n+1)}\right)}{n+1}=\frac{1}{2 \pi} \int_{0}^{2 \pi} f\left(e^{i \theta}\right) d \theta .
$$

In general, one should compare Theorem 2 with the quadrature formulas for the Poisson integrals as obtained recently by Bultheel et al. [3]. It is essentially (though not exactly) equivalent to the convergence result in [3] (via suitable transformations and modifications). We will give a proof of Theorem 2 that is independent of [3] to indicate a different and direct approach.

Walsh's Theorem holds for all $f$ that are Riemann integrable on $\mathbf{T}$, while in Theorem 1 we only managed to obtain the case when $f$ is assumed continuous on $\mathbf{T}$. With more restrictive conditions on the rate in which the poles are allowed to approach the unit circle $\mathbf{T}$, we do have an extension of Walsh's theorem for all Riemann integrable functions as follows. 
Theorem 3. Assume that

$$
\lim _{n \rightarrow \infty} \sigma_{n} \delta_{n}=\infty
$$

If $f$ is Riemann integrable on $\mathbf{T}$, then

$$
\lim _{n \rightarrow \infty} \sum_{k=0}^{n} \frac{f\left(z_{n, k}\right)}{\left|B_{n}^{\prime}\left(z_{n, k}\right)\right|}=\frac{1}{2 \pi} \int_{0}^{2 \pi} f\left(e^{i \theta}\right) d \theta
$$

and

$$
\lim _{n \rightarrow \infty} R_{n}(f ; z)=f_{\mathbf{D}}(z)
$$

for $z \in \mathbf{D}$, and the convergence is uniform on any compact subset of $\mathbf{D}$.

Condition (3) still allows $\alpha_{n}$ to approach $\mathbf{T}$ but is more restrictive on its rate of convergence. For example, $\alpha_{n}=1-1 / n^{\delta}, n=1,2, \ldots$, with $\delta \in[0,1 / 2)$ satisfy (3). Since $\sigma_{n}>\sigma_{n} \delta_{n}$, (3) implies $\lim _{n \rightarrow \infty} \sigma_{n}=\infty$, which is what we assumed in Theorem 1. It is not clear whether (3) can be replaced by the weaker condition $\lim _{n \rightarrow \infty} \sigma_{n}=\infty$ in the assumption of Theorem 3 .

Next, we present the $L_{2}$ convergence of $\left\{R_{n}(f ; \cdot)\right\}$ for $f \in A(\mathbf{D})$, which generalizes the result of Walsh and Sharma [26] and that of Lozinski [15] for $p=2$ in (1).

Theorem 4. If $\lim _{n \rightarrow \infty} \sigma_{n}=\infty$, then

$$
\lim _{n \rightarrow \infty} \int_{\mathbf{T}}\left|R_{n}(f ; z)-f(z)\right|^{2}|d z|=0,
$$

for every $f \in A(\mathbf{D})$.

Similar to the polynomial case, this implies a rational version of Fejer's result mentioned in the Introduction.

The truths of Theorems 1 and 4 not only reveal a clear analogy between polynomial and rational interpolations on the unit circle $\mathbf{T}$, but also implicitly indicate how the extension from polynomial cases to rational cases can be carried out. For example, the well-known result of Walsh, the equi-convergence theorem [25, Theorem 1, p. 153], is extended for rational interpolation in the following result.

Theorem 5. Assume that the set $\left\{\alpha_{0}, \alpha_{1}, \ldots\right\}$ has no limit point on $\mathbf{T}$ and its closure $\operatorname{cls}\left(\left\{\alpha_{0}, \alpha_{1}, \ldots\right\}\right)$ does not separate the plane $\mathbf{C}$. If

$$
\lim _{n \rightarrow \infty}\left|B_{n}(z)\right|^{1 / n}=\psi(z) \not \equiv \text { constant }
$$

holds locally uniformly on $\mathbf{C} \backslash\left(\mathbf{D} \cup \overline{\left\{1 / \overline{\alpha_{0}}, 1 / \overline{\alpha_{1}}, \ldots\right\}}\right)$, then

$$
\lim _{n \rightarrow \infty}\left[R_{n}(f ; z)-r_{n}(f ; z)\right]=0 \quad \text { for } \quad \psi(z)<\rho^{2}
$$

where $f$ is assumed analytic for $\psi(z)<\rho(\rho>1)$ and $r_{n}(f ; z)$ is the least-square approximation to $f$ out of $\mathcal{R}_{n}$, i.e.,

$$
\int_{\mathbf{T}}\left|f(z)-r_{n}(f ; z)\right|^{2}|d z|=\min _{r \in \mathcal{R}_{n}} \int_{\mathbf{T}}|f(z)-r(z)|^{2}|d z| .
$$


This result is analogous to one proved by Saff and Sharma in [20] for a different rational system in which poles are the roots of $z^{n}-r^{n}=0$ for some $r>1$ (and so the poles are not from a single sequence as we have assumed in this paper).

In the assumption of Theorem 5, the required range of $z$ implied by (6) can be made smaller. We have used the stronger assumption for simplicity. When $\left\{\alpha_{n}\right\}_{n=0}^{\infty}$ is cyclic, i.e.,

$$
\alpha_{k m+r}=\alpha_{r}, \quad r=0,1, \ldots, m-1, \quad k=1,2, \ldots,
$$

for some $m \geq 1$, then (6) is satisfied as

$$
\lim _{n \rightarrow \infty}\left|B_{n}(z)\right|^{1 / n}=\left|\prod_{k=0}^{m-1} \frac{z-\alpha_{k}}{1-\overline{\alpha_{k}} z}\right|^{1 / m} .
$$

We refer to Walsh's discussion in [25, $\S 99.4,9.5$, in particular, p. 236] for more properties of $\psi(z)$. Here we only mention the following fact that will be needed in our proof: The locus $\gamma\left(\rho_{1}\right):=\left\{z: \psi(z)=\rho_{1}\right\}$ is rectifiable and, for $1<\rho_{1}<\rho, \gamma\left(\rho_{1}\right)$, lies in $\{z: \psi(z)<\rho\}$ and is exterior to $\mathbf{T}$.

Note that for $f$ analytic on $\overline{\mathbf{D}}$, the least square approximation to $f$ out of $\mathcal{P}_{n}$ is the same as the $n$th Taylor polynomial of $f$ about $z=0$. As all our theorems imply the corresponding known results for polynomial interpolation, Theorem 5 will be given the following form when we let $\alpha_{n}=0$ for all $n=0,1,2, \ldots$.

Corollary 6. If $f$ is analytic for $|z|<\rho(\rho>1)$, then

$$
\lim _{n \rightarrow \infty}\left[L_{n}(f ; z)-s_{n}(f ; z)\right]=0 \quad \text { for } \quad|z|<\rho^{2}
$$

where $L_{n}(f ; z)$ is the polynomial interpolating $f$ at the $n+1$ st roots of unity, and $s_{n}(f ; z)$ is the Taylor polynomial of degree $n$ of $f$ about $z=0$.

This is the celebrated Walsh's equi-convergence theorem mentioned above. This theorem has been much extended in recent years; for the latest results, see $[4,5,17,18]$ and the bibliographies therein.

\section{Auxiliary results}

We collect some known results and prove some new ones that are needed in the proofs of the theorems.

Lemma 1. For $k=0,1, \ldots, n$, we have $z_{n, k} B_{n}^{\prime}\left(z_{n, k}\right)=\left|B_{n}^{\prime}\left(z_{n, k}\right)\right|$.

Proof. A straightforward calculation (cf. (i) of [14, Lemma 1]) shows that

$$
\frac{z B_{n}^{\prime}(z)}{B_{n}(z)}=\left|B_{n}^{\prime}(z)\right| \quad \text { for all } \quad z \in \mathbf{T} \text {. }
$$

Letting $z=z_{n, k}$ yields the desired equality.

Lemma 2. For $n=0,1,2, \ldots$, we have

$$
\sum_{k=0}^{n} \frac{1}{\left|B_{n}^{\prime}\left(z_{n, k}\right)\right|}=\frac{1-\left|B_{n}(0)\right|^{2}}{\left|1-B_{n}(0)\right|^{2}}
$$


Proof. Let $f_{I}(z):=1-\overline{B_{n}(0)} B_{n}(z)$. Then $f_{I} \in \mathcal{R}_{n}$ and

$$
f\left(z_{n, k}\right)=1-\overline{B_{n}(0)} B_{n}\left(z_{n, k}\right)=1-\overline{B_{n}(0)}, \quad k=0,1, \ldots, n .
$$

So, by the uniqueness of the interpolating rational function,

$$
f_{I}(z)=R_{n}\left(f_{I} ; z\right)=\sum_{k=0}^{n} \frac{\left(1-\overline{B_{n}(0)}\right)\left(B_{n}(z)-1\right)}{B_{n}^{\prime}\left(z_{n, k}\right)\left(z-z_{n, k}\right)} .
$$

Hence, we have the identity

$$
\sum_{k=0}^{n} \frac{1}{B_{n}^{\prime}\left(z_{n, k}\right)\left(z-z_{n, k}\right)}=\frac{1-\overline{B_{n}(0)} B_{n}(z)}{\left(1-\overline{B_{n}(0)}\right)\left(B_{n}(z)-1\right)} .
$$

Letting $z=0$ gives us

$$
\sum_{k=0}^{n} \frac{1}{z_{n, k} B_{n}^{\prime}\left(z_{n, k}\right)}=\frac{1-\left|B_{n}(0)\right|^{2}}{\left|1-B_{n}(0)\right|^{2}}
$$

which, by Lemma 1 , is the identity we need to verify.

Lemma 3. The linear span of $\mathcal{R} \cup \mathcal{R}^{*}$ is dense in $C(\mathbf{T})$ if and only if $\lim _{n \rightarrow \infty} \sigma_{n}=\infty$.

Proof. This follows from an application of a result in [1, Addendum A, §2, p. 244]. It can be proved by the same method.

Lemma 4. For $k=0,1, \ldots, n-1$, there exist numbers $\xi_{k}, \zeta_{k} \in\left(\theta_{n, k}, \theta_{n, k+1}\right)$ such that

$$
\theta_{n, k+1}-\theta_{n, k}=\frac{2 \pi}{\left|B_{n}^{\prime}\left(e^{i \xi_{k}}\right)\right|}
$$

and

$$
2 \pi-\left(\theta_{n, k+1}-\theta_{n, k}\right)\left|B_{n}^{\prime}\left(z_{n, k}\right)\right|=\frac{2 \pi \gamma_{n}^{\prime \prime}\left(\zeta_{k}\right)\left(\theta_{n, k+1}-\zeta_{k}\right)}{\gamma_{n}^{\prime}\left(\zeta_{k}\right)}
$$

where $\gamma_{n}(\theta)$ is defined as a continuous function satisfying

$$
B_{n}\left(e^{i \theta}\right)=e^{i \gamma_{n}(\theta)}, \quad \theta \in[0,2 \pi] .
$$

It turns out that, such a function $\gamma_{n}$ is uniquely determined (up to an additional constant) and continuously differentiable on $[0,2 \pi]$. Indeed, we have (see, for example, [13])

$$
\gamma_{n}^{\prime}(\theta)=\left|B_{n}^{\prime}\left(e^{i \theta}\right)\right|=\sum_{k=0}^{n} \frac{1-\left|\alpha_{k}\right|^{2}}{\left|e^{i \theta}-\alpha_{k}\right|^{2}}=\sum_{k=0}^{n} \frac{1-r_{k}^{2}}{1-2 r_{k} \cos \left(\theta-\omega_{k}\right)+r_{k}^{2}}
$$

where $\alpha_{k}=r_{k} e^{i \omega_{k}}$ with $r_{k}>0, k=0,1, \ldots$ From (9), we can estimate $\gamma^{\prime}$ as follows:

$$
\gamma^{\prime}(\theta)=\left|B_{n}^{\prime}\left(e^{i \theta}\right)\right| \geq \sum_{k=0}^{n} \frac{1-r_{k}}{1+r_{k}}>\frac{1}{2} \sum_{k=0}^{n}\left(1-r_{k}\right)=\frac{1}{2} \sigma_{n} .
$$


Proof of Lemma 4. Note that $\gamma_{n}\left(\theta_{n, k+1}\right)-\gamma_{n}\left(\theta_{n, k}\right)=2 \pi$. Now, by the mean value theorem, we can write, for some $\xi_{k} \in\left(\theta_{n, k}, \theta_{n, k+1}\right)$,

$$
2 \pi=\gamma_{n}\left(\theta_{n, k+1}\right)-\gamma_{n}\left(\theta_{n, k}\right)=\gamma_{n}^{\prime}\left(\xi_{k}\right)\left(\theta_{n, k+1}-\theta_{n, k}\right),
$$

which, by (9), implies (7).

To verify (8), let

$$
g(\theta):=-\gamma_{n}\left(\theta_{n, k+1}\right)+\gamma_{n}(\theta)+\left(\theta_{n, k+1}-\theta\right) \gamma_{n}^{\prime}(\theta) .
$$

Then,

$$
g\left(\theta_{n, k+1}\right)-g\left(\theta_{n, k}\right)=2 \pi-\left(\theta_{n, k+1}-\theta_{n, k}\right)\left|B_{n}^{\prime}\left(z_{n, k}\right)\right|
$$

and

$$
g^{\prime}(\theta)=\gamma_{n}^{\prime \prime}(\theta)\left(\theta_{n, k+1}-\theta\right) .
$$

Then, by the generalized Cauchy mean value theorem, there exists a number $\zeta_{k} \in$ $\left(\theta_{n, k}, \theta_{n, k+1}\right)$ such that

$$
\frac{g\left(\theta_{n, k+1}\right)-g\left(\theta_{n, k}\right)}{\gamma_{n}\left(\theta_{n, k+1}\right)-\gamma_{n}\left(\theta_{n, k}\right)}=\frac{g^{\prime}\left(\zeta_{k}\right)}{\gamma_{n}^{\prime}\left(\zeta_{k}\right)}
$$

Now, equation (8) follows.

Lemma 5. For $\zeta, z \in \mathbf{C}$, we have

$$
\sum_{j=0}^{n} \frac{B_{n}(z)}{B_{n}^{\prime}\left(\alpha_{j}\right)\left(z-\alpha_{j}\right)\left(1-\bar{\zeta} \alpha_{j}\right)}= \begin{cases}\frac{z B_{n}^{\prime}(z)}{B_{n}(z)}, & \text { if } z=\frac{1}{\bar{\zeta}} \\ \frac{1-\overline{B_{n}(\zeta)} B_{n}(z)}{1-\bar{\zeta} z}, & \text { otherwise }\end{cases}
$$

Proof. First, assume $\zeta \in \mathbf{D}$. Then, note that

$$
\frac{1-\overline{B_{n}(\zeta)} B_{n}(z)}{1-\bar{\zeta} z}
$$

is the rational function from $\mathcal{R}_{n}$ that interpolates $f_{\zeta}(z)=1 /(1-\bar{\zeta} z)$ at $z=$ $\alpha_{0}, \alpha_{1}, \ldots, \alpha_{n}$. Thus, by Lagrange's interpolation formula, we have

$$
\frac{1-\overline{B_{n}(\zeta)} B_{n}(z)}{1-\bar{\zeta} z}=\sum_{k=0}^{n} \frac{B_{n}(z)}{B_{n}^{\prime}\left(z_{n, k}\right)\left(z-\alpha_{k}\right)\left(1-\bar{\zeta} \alpha_{k}\right)}
$$

Observe that both sides of (11) are rational functions of the same type in $z$ and, after taking the complex conjugate of both sides in (11), in $\zeta$. So, in general, the equality in (11) holds as long as $\bar{\zeta} z \neq 1$.

Finally, the case when $z=1 / \bar{\zeta}$ can be handled by first taking the limit as $z \rightarrow 1 / \bar{\zeta}$ in (11) and then write $\zeta$ as $1 / \bar{z}$.

We will need the system of functions from $\mathcal{R}$ given by

$$
\varphi_{n}(z)=\frac{\sqrt{1-\left|\alpha_{n}\right|^{2}}}{z-\alpha_{n}} B_{n}(z), \quad n=0,1,2, \ldots
$$

This system was probably introduced (with a constant multiplier of modulus 1 ) first by Takenaka in [23] and Malmquist in [16]. See also [8] and [25, p. 224]. It is orthogonal with respect to the uniform measure $d \theta /(2 \pi)$ on the unit circle $\mathbf{T}$ (with $z=e^{i \theta}$ ). We collect some properties of $\left\{\varphi_{n}\right\}$ in the following lemma. 
Lemma 6. (i) For $n=0,1, \ldots,\left\{\varphi_{k}(z)\right\}_{k=0}^{n} \subseteq \mathcal{R}_{n}$ and, $j, k=0,1,2, \ldots$,

$$
\frac{1}{2 \pi} \int_{z \in \mathbf{T}} \varphi_{j}(z) \overline{\varphi_{k}(z)}|d z|= \begin{cases}0, & j \neq k, \\ 1, & j=k .\end{cases}
$$

(ii) (Christoffel-Darboux formula for $\left\{\varphi_{n}\right\}$ ) For all $\zeta, z \in \mathbf{C}$, we have

$$
\sum_{k=0}^{n} \overline{\varphi_{k}(\zeta)} \varphi_{k}(z)=\frac{1-\overline{B_{n}(\zeta)} B_{n}(z)}{1-\bar{\zeta} z}
$$

Proof. A proof of equation (12) can be found in [25, p. 227]. The identity (13) is well known in the literature (see [8]). It is a special case of the more general ChristoffelDarboux formula established for rational functions orthogonal on the unit circle. See, for example, [2].

Our next result is interesting in its own right. It generalizes a relation first observed by Walsh and Sharma [26, Formula (16)] on polynomials.

Lemma 7. For $p, q=0,1, \ldots, n$, we have

$$
\frac{1}{2 \pi} \int_{\mathbf{T}} \frac{B_{n}(z)-1}{z-z_{n, p}} \overline{\left(\frac{B_{n}(z)-1}{z-z_{n, q}}\right)}|d z|= \begin{cases}0, & p \neq q \\ \left|B_{n}^{\prime}\left(z_{n, p}\right)\right|, & p=q .\end{cases}
$$

Proof. Let

$$
I(p, q):=\frac{1}{2 \pi} \int_{\mathbf{T}} \frac{B_{n}(z)-1}{z-z_{n, p}} \overline{\left(\frac{B_{n}(z)-1}{z-z_{n, q}}\right)}|d z| .
$$

We claim that

$$
I(p, q)=z_{n, q} \overline{z_{n, p}} \overline{\sum_{j=0}^{n} \frac{B_{n}\left(z_{n, p}\right)}{B_{n}^{\prime}\left(\alpha_{j}\right)\left(z_{n, p}-\alpha_{j}\right)\left(1-\overline{z_{n, q}} \alpha_{j}\right)}} .
$$

Assuming the truth of (14), we see that Lemma 5 implies $I(p, q)=0$ when $p \neq q$, and Lemmas 1 and 5 infer that $I(p, q)=\left|B_{n}^{\prime}\left(z_{n, p}\right)\right|$. Therefore, the lemma follows from (14).

Now, let us verify (14). We need the orthonormal basis $\left\{\varphi_{k}\right\}_{k=0}^{n}$ in $\mathcal{R}_{n}$ introduced above. Since

we can write

$$
\frac{B_{n}(z)-1}{z-z_{n, p}}, \quad \frac{B_{n}(z)-1}{z-z_{n, q}} \in \mathcal{R}_{n}
$$

$$
\frac{B_{n}(z)-1}{z-z_{n, p}}=\sum_{k=0}^{n} a_{k} \varphi_{k}(z) \quad \text { and } \quad \frac{B_{n}(z)-1}{z-z_{n, q}}=\sum_{k=0}^{n} b_{k} \varphi_{k}(z)
$$

for some $\left\{a_{k}\right\}_{k=0}^{n}$ and $\left\{b_{k}\right\}_{k=0}^{n}$. Then, for $p, q=0,1, \ldots, n$, by (12) in Lemma 6, we have $I(p, q)=\sum_{k=0}^{n} a_{k} \overline{b_{k}}$. Now,

$$
\begin{aligned}
\overline{a_{k}} & =\overline{\frac{1}{2 \pi} \int_{\mathbf{T}} \frac{B_{n}(z)-1}{z-z_{n, p}} \overline{\varphi_{k}(z)}|d z|}=\frac{1}{2 \pi i} \int_{\mathbf{T}} \frac{\left(1-B_{n}(z)\right) \varphi_{k}(z)}{B_{n}(z)\left(1-\overline{z_{n, p}} z\right)} d z \\
& =\sum_{j=0}^{n} \frac{\varphi_{k}\left(\alpha_{j}\right)}{\overline{B_{n}^{\prime}\left(\alpha_{j}\right)\left(1-\overline{z_{n, p}} \alpha_{j}\right)}}
\end{aligned}
$$


by the residue theorem. Similarly,

$$
\overline{b_{k}}=\sum_{j=0}^{n} \frac{\varphi_{k}\left(\alpha_{j}\right)}{B_{n}^{\prime}\left(\alpha_{j}\right)\left(1-\overline{z_{n, q}} \alpha_{j}\right)}
$$

Hence,

$$
\begin{aligned}
I(p, q) & =\sum_{k=0}^{n} \sum_{j_{1}=0}^{n} \frac{\overline{\varphi_{k}\left(\alpha_{j_{1}}\right)}}{\overline{B_{n}^{\prime}\left(\alpha_{j_{1}}\right)}\left(1-z_{n, p} \overline{\alpha_{j_{1}}}\right)} \sum_{j_{2}=0}^{n} \frac{\varphi_{k}\left(\alpha_{j_{2}}\right)}{\overline{B_{n}^{\prime}\left(\alpha_{j_{2}}\right)\left(1-\overline{z_{n, q}} \alpha_{j_{2}}\right)}} \\
& =\sum_{j_{1}, j_{2}=0}^{n} \overline{\overline{B_{n}^{\prime}\left(\alpha_{j_{1}}\right)} B_{n}^{\prime}\left(\alpha_{j_{2}}\right)\left(1-z_{n, p} \overline{\alpha_{j_{1}}}\right)\left(1-\overline{z_{n, q}} \alpha_{j_{2}}\right)} \sum_{k=0}^{n} \overline{\varphi_{k}\left(\alpha_{j_{1}}\right)} \varphi_{k}\left(\alpha_{j_{2}}\right) \\
& =\sum_{j_{1}, j_{2}=0}^{n} \overline{\overline{B_{n}^{\prime}\left(\alpha_{j_{1}}\right)} B_{n}^{\prime}\left(\alpha_{j_{2}}\right)\left(1-z_{n, p} \overline{\alpha_{j_{1}}}\right)\left(1-\overline{z_{n, q}} \alpha_{j_{2}}\right)} \frac{1-\overline{B_{n}\left(\alpha_{j_{1}}\right)} B_{n}\left(\alpha_{j_{2}}\right)}{1-\overline{\alpha_{j_{1}}} \alpha_{j_{2}}}
\end{aligned}
$$

by (13) in Lemma 6 . So,

$$
\begin{aligned}
I(p, q) & =\sum_{j_{1}, j_{2}=0}^{n} \frac{1}{\overline{B_{n}^{\prime}\left(\alpha_{j_{1}}\right)} B_{n}^{\prime}\left(\alpha_{j_{2}}\right)\left(1-z_{n, p} \overline{\alpha_{j_{1}}}\right)\left(1-\overline{z_{n, q}} \alpha_{j_{2}}\right)} \frac{1}{1-\overline{\alpha_{j_{1}}} \alpha_{j_{2}}} \\
& =\sum_{j_{1}=0}^{n} \frac{1}{\overline{B_{n}^{\prime}\left(\alpha_{j_{1}}\right)}\left(1-z_{n, p} \overline{\alpha_{j_{1}}}\right)}\left(\sum_{j_{2}=0}^{n} \frac{1}{\left.\overline{B_{n}^{\prime}\left(\alpha_{j_{2}}\right)\left(1-\overline{z_{n, q}} \alpha_{j_{2}}\right)\left(1-\overline{\alpha_{j_{1}}} \alpha_{j_{2}}\right)}\right) .}\right.
\end{aligned}
$$

Now, note that

$$
\sum_{j_{2}=0}^{n} \frac{1}{B_{n}^{\prime}\left(\alpha_{j_{2}}\right)\left(1-\overline{z_{n, q}} \alpha_{j_{2}}\right)\left(1-\overline{\alpha_{j_{1}}} \alpha_{j_{2}}\right)}=z_{n, q} \sum_{j_{2}=0}^{n} \frac{B_{n}\left(z_{n, q}\right)}{B_{n}^{\prime}\left(\alpha_{j_{2}}\right)\left(z_{n, q}-\alpha_{j_{2}}\right)\left(1-\overline{\alpha_{j_{1}}} \alpha_{j_{2}}\right)}
$$

which, by using Lemma 5 with $z=z_{n, q}$ and $\zeta=\alpha_{j_{1}}$, is equal to

$$
z_{n, q} \frac{1-\overline{B_{n}\left(\alpha_{j_{1}}\right)} B_{n}\left(z_{n, q}\right)}{1-\overline{\alpha_{j_{1}}} z_{n, q}}=\frac{z_{n, q}}{1-\overline{\alpha_{j_{1}}} z_{n, q}}
$$

Thus,

$$
\begin{aligned}
I(p, q) & =\sum_{j_{1}=0}^{n} \frac{1}{\overline{B_{n}^{\prime}\left(\alpha_{j_{1}}\right)}\left(1-z_{n, p} \overline{\alpha_{j_{1}}}\right)} \frac{z_{n, q}}{1-\overline{\alpha_{j_{1}}} z_{n, q}} \\
& =z_{n, q} \overline{z_{n, p}} \sum_{j=0}^{n} \frac{B_{n}\left(z_{n, p}\right)}{\overline{B_{n}^{\prime}\left(\alpha_{j}\right)\left(z_{n, p}-\alpha_{j}\right)\left(1-\overline{z_{n, q}} \alpha_{j}\right)}}
\end{aligned}
$$

which is (14). This completes the proof. 


\section{Proofs of the main theorems}

We prove Theorem 2 first since our proof of Theorem 1 is based on it.

Proof of Theorem 2. We first claim that

$$
\lim _{n \rightarrow \infty} \int_{\mathbf{T}} r d \nu_{n}=\frac{1}{2 \pi} \int_{\mathbf{T}} r(z)|d z|
$$

for every $r \in \mathcal{R} \cup \mathcal{R}^{*}$. This is a consequence of the following identity.

$$
\sum_{k=0}^{n} \frac{f\left(z_{n, k}\right)}{\left|B_{n}^{\prime}\left(z_{n, k}\right)\right|}=\frac{1}{2 \pi\left(1-B_{n}(0)\right)} \int_{\mathbf{T}} f(z)|d z|
$$

for every $f \in \mathcal{R}_{n}$. Indeed, for $f \in \mathcal{R}_{n}$, we have $f(z)=R_{n}(f ; z)$. So, by Lemma 1 ,

$$
f(0)=R_{n}(f ; 0)=\sum_{k=0}^{n} \frac{f\left(z_{n, k}\right)\left(1-B_{n}(0)\right)}{z_{n, k} B_{n}^{\prime}\left(z_{n, k}\right)}=\sum_{k=0}^{n} \frac{f\left(z_{n, k}\right)\left(1-B_{n}(0)\right)}{\left|B_{n}^{\prime}\left(z_{n, k}\right)\right|} .
$$

This, on writing $f(0)=\int_{\mathbf{T}} f(z)|d z|$, implies equation (16). Since $\mathcal{R}_{n} \subset \mathcal{R}_{n+1}$, we let $n \rightarrow \infty$ in (16) to obtain (15) for $r \in \mathcal{R}$ by using the fact that $\lim _{n \rightarrow \infty} B_{n}(0)=0$. Next, by taking the complex conjugate of both sides of (15), we see that (15) holds for $f \in \mathcal{R}_{n}^{*}$ as well.

Now, from Lemma 2, it follows that

$$
\int_{\mathbf{T}} d \nu_{n}=\frac{1-\left|B_{n}(0)\right|^{2}}{\left|1-B_{n}(0)\right|^{2}} \leq \frac{2}{1-\left|B_{n}(0)\right|} \leq \frac{2}{1-\left|\alpha_{0}\right|}
$$

for $n=0,1,2, \ldots$. Thus $\left\{d \nu_{n}\right\}$ is compact in the weak-star topology. Let $d \nu$ be any weak-star limit of $\left\{d \nu_{n}\right\}$ and

$$
\lim _{\substack{n \rightarrow \infty \\ n \in \Gamma}} \int_{\mathbf{T}} f d \nu_{n}=\int_{\mathbf{T}} f d \nu
$$

for every $f \in C(\mathbf{T})$. In view of (15), this means

$$
\int_{\mathbf{T}} r d \nu=\frac{1}{2 \pi} \int_{\mathbf{T}} r(z)|d z|
$$

for every $r \in \mathcal{R} \cup \mathcal{R}^{*}$. Since, by Lemma 3 , the linear span of $\mathcal{R} \cup \mathcal{R}^{*}$ is dense in $C(\mathbf{T})$, then $d \nu(\theta)=d \theta /(2 \pi)$. Therefore, the whole sequence converges to $d \theta /(2 \pi)$.

Proof of Theorem 1. Let $f \in C(\mathbf{T})$. By using Lemma 1, we can write

$$
R_{n}(f ; z)=\sum_{k=0}^{n} \frac{f\left(z_{n, k}\right)\left(B_{n}(z)-1\right)}{B_{n}^{\prime}\left(z_{n, k}\right)\left(z-z_{n, k}\right)}=\left(1-B_{n}(z)\right) \sum_{k=0}^{n} \frac{z_{n, k} f\left(z_{n, k}\right)}{z_{n, k}-z} \frac{1}{\left|B_{n}^{\prime}\left(z_{n, k}\right)\right|}
$$

For each fixed $z \in \mathbf{D}$, we have $\lim _{n \rightarrow \infty} B_{n}(z)=0$ and that

$$
\frac{t f(t)}{t-z}
$$

is continuous for $t \in[0,2 \pi]$. So, using Theorem 2 , we obtain

$$
\lim _{n \rightarrow \infty} R_{n}(f ; z)=\frac{1}{2 \pi} \int_{\mathbf{T}} \frac{t f(t)}{t-z}|d t|=\frac{1}{2 \pi} \int_{\mathbf{T}} \frac{f(t)}{t-z} d t
$$

which is $f_{\mathbf{D}}(z)$. 
Proof of Theorem 3. We show that

$$
\left|\frac{1}{\left|B_{n}^{\prime}\left(z_{n, k}\right)\right|}-\frac{\theta_{n, k+1}-\theta_{n, k}}{2 \pi}\right| \leq \frac{8 \pi}{\left|B_{n}^{\prime}\left(z_{n, k}\right)\right| \sigma_{n} \delta_{n}} .
$$

Dividing both sides of (8) by $2 \pi\left|B_{n}^{\prime}\left(z_{n, k}\right)\right|$, we have

$$
\left|\frac{1}{\left|B_{n}^{\prime}\left(z_{n, k}\right)\right|}-\frac{\theta_{n, k+1}-\theta_{n, k}}{2 \pi}\right|=\frac{\left|\gamma^{\prime \prime}\left(\zeta_{k}\right)\left(\theta_{n, k+1}-\zeta_{k}\right)\right|}{\left|B_{n}^{\prime}\left(z_{n, k}\right)\right| \gamma^{\prime}\left(\zeta_{k}\right)}
$$

Now, note that

$$
\left|\frac{\gamma_{n}^{\prime \prime}\left(\zeta_{k}\right)}{\gamma_{n}^{\prime}\left(\zeta_{k}\right)}\right| \leq \frac{\sum_{j=0}^{n} c_{j} \frac{2 r_{j}\left|\sin \left(\zeta_{k}-\omega_{j}\right)\right|}{1-2 r_{j} \cos \left(\zeta_{k}-\omega_{j}\right)+r_{j}^{2}}}{\sum_{j=0}^{n} c_{j}}
$$

with

$$
c_{j}:=\frac{1-r_{j}^{2}}{1-2 r_{j} \cos \left(\zeta_{k}-\omega_{j}\right)+r_{j}^{2}}, \quad j=0,1, \ldots, n .
$$

Note that, $c_{j}>0, j=0,1, \ldots, n$, and, for $0 \leq r<1$,

$$
\max _{\theta \in[0,2 \pi]} \frac{2 r|\sin (\theta)|}{1-2 r \cos (\theta)+r^{2}}=\frac{2 r}{1-r^{2}} .
$$

So, we have

$$
\left|\frac{\gamma_{n}^{\prime \prime}\left(\zeta_{k}\right)}{\gamma_{n}^{\prime}\left(\zeta_{k}\right)}\right| \leq \max _{0 \leq j \leq n} \frac{2 r_{j}}{1-r_{j}^{2}}<\frac{2}{\delta_{n}}
$$

On the other hand, by (7) in Lemma 4 and (10), we have

$$
\left|\theta_{n, k+1}-\zeta_{k}\right| \leq\left|\theta_{n, k+1}-\theta_{n, k}\right|=\frac{2 \pi}{\left|B_{n}^{\prime}\left(e^{i \xi_{k}}\right)\right|} \leq \frac{2 \pi}{\frac{1}{2} \sigma_{n}}=\frac{4 \pi}{\sigma_{n}}
$$

Using (19) and (20) in (18), we obtain (17).

To prove (4), we use (17) and compare the sum

$$
\sum_{k=0}^{n} \frac{f\left(z_{n, k}\right)}{\left|B_{n}^{\prime}\left(z_{n, k}\right)\right|}
$$

with

$$
\frac{1}{2 \pi} \sum_{k=0}^{n} f\left(e^{i \theta_{n, k}}\right)\left(\theta_{n, k+1}-\theta_{n, k}\right)
$$


a Riemann sum of $f$ on $\mathbf{T}$. We have, with $M_{f}$ denoting a upper bound of $f$ on $\mathbf{T}$, by using (17) and Lemma 2,

$$
\begin{aligned}
& \left|\sum_{k=0}^{n} \frac{f\left(z_{n, k}\right)}{\left|B_{n}^{\prime}\left(z_{n, k}\right)\right|}-\frac{1}{2 \pi} \sum_{k=0}^{n} f\left(e^{i \theta_{n, k}}\right)\left(\theta_{n, k+1}-\theta_{n, k}\right)\right| \\
& \quad \leq \sum_{k=0}^{n}\left|f\left(z_{n, k}\right)\right|\left|\frac{1}{\left|B_{n}^{\prime}\left(z_{n, k}\right)\right|}-\frac{\theta_{n, k+1}-\theta_{n, k}}{2 \pi}\right| \\
& \quad \leq M_{f} \sum_{k=0}^{n} \frac{8 \pi}{\left|B_{n}^{\prime}\left(z_{n, k}\right)\right| \sigma_{n} \delta_{n}}=\frac{8 \pi M_{f}\left(1-\left|B_{n}(0)\right|^{2}\right)}{\sigma_{n} \delta_{n}\left(\left|1-B_{n}(0)\right|^{2}\right)}
\end{aligned}
$$

which goes to the limit zero by the assumption (3). This proves (4).

The proof of (5) is obtained from (4) in the same way as that of Theorem 1 is obtained from Theorem 2 .

Proof of Theorem 4. Since $f \in A(\mathbf{D})$, by Mergelyan's theorem ([10, Theorem 1, p. 97]), there exists a sequence of polynomials (the arithmetic means of the partial sums of the Taylor series of $f$ about $z=0$ ) that converges uniformly to $f$ on $\overline{\mathbf{D}}$. By using the argument in [1, Addendum A, pp. 243-246], we can see that each monomial $z^{k}$ $(k \geq 0)$ can be approximated by rational functions from $\mathcal{R}$ as closely as we please. Therefore, for $\varepsilon>0$, there exists a function $r \in \mathcal{R}$ such that

$$
\max _{z \in \overline{\bar{D}}}|f(z)-r(z)|<\varepsilon
$$

Note that $R_{n}(r ; z)=r(z)$ for all $n$ large enough. Now we have, for $n$ large enough,

$$
\begin{aligned}
\int_{\mathbf{T}} \mid & R_{n}(f ; z)-\left.f(z)\right|^{2}|d z|=\int_{\mathbf{T}}\left|R_{n}(f-r ; z)+r(z)-f(z)\right|^{2}|d z| \\
& \leq 2\left\{\int_{\mathbf{T}}\left|R_{n}(f-r ; z)\right|^{2}|d z|+\int_{\mathbf{T}}|r(z)-f(z)|^{2}|d z|\right\} \\
& \leq 2 \int_{\mathbf{T}}\left|R_{n}(f-r ; z)\right|^{2}|d z|+4 \pi \varepsilon^{2} .
\end{aligned}
$$

The integral in the last expression can be expanded as

$$
\sum_{p, q=0}^{n} \frac{f\left(z_{n, p}\right)-r\left(z_{n, p}\right)}{B_{n}^{\prime}\left(z_{n, p}\right)} \overline{\left[\frac{f\left(z_{n, q}\right)-r\left(z_{n, q}\right)}{B_{n}^{\prime}\left(z_{n, q}\right)}\right]} \int_{\mathbf{T}} \frac{B_{n}(z)-1}{z-z_{n, p}} \overline{\left(\frac{B_{n}(z)-1}{z-z_{n, q}}\right)}|d z|,
$$

which, according to Lemma 7 , is equal to

$$
\sum_{k=0}^{n} \frac{2 \pi\left|f\left(z_{n, k}\right)-r\left(z_{n, k}\right)\right|^{2}}{\left|B_{n}^{\prime}\left(z_{n, k}\right)\right|}
$$

Now, combining the above estimates and using Lemma 2, we have

$$
\int_{\mathbf{T}}\left|R_{n}(f ; z)-f(z)\right|^{2}|d z| \leq \frac{4 \pi \varepsilon^{2}\left(1-\left|B_{n}(0)\right|^{2}\right)}{\left|1-B_{n}(0)\right|^{2}}+4 \pi \varepsilon^{2} \leq \frac{12 \pi \varepsilon^{2}}{1-\left|B_{n}(0)\right|} .
$$

Thus, by letting $n \rightarrow \infty$, we obtain

$$
0 \leq \limsup _{n \rightarrow \infty} \int_{\mathbf{T}}\left|R_{n}(f ; z)-f(z)\right|^{2}|d z| \leq 12 \pi \varepsilon^{2} .
$$

This implies the desired result. 
Proof of Theorem 5. By Hermite's formula, for each $n=1,2, \ldots$,

$$
R_{n}(f ; z)=\frac{1}{2 \pi} \int_{\gamma} \frac{f(\zeta)\left(B_{n}(\zeta)-B_{n}(z)\right)}{\left(B_{n}(\zeta)-1\right)(\zeta-z)} d \zeta
$$

for all $z \in \operatorname{Int}(\gamma)$, the interior of $\gamma$ where $\gamma$ is a Jordan curve in $\{z: \psi(z)<\rho\}$ that surrounds $\left\{z_{n, 0}, z_{n, 1}, \ldots, z_{n, n}\right\} \subseteq \mathbf{T}$. On the other hand, by [25, Lemmas I and II on p. 225, Theorem 2 on p. 227], the least square approximation $r_{n}(f ; z)$ is the same as the rational function from $\mathcal{R}_{n}$ that interpolates $f$ at $\alpha_{0}, \alpha_{1}, \ldots, \alpha_{n}$. (For repeated points, $r_{n}$ interpolates $f$ and its derivatives.) Again, by Hermite's formula,

$$
r_{n}(f ; z)=\frac{1}{2 \pi} \int_{\gamma} \frac{f(\zeta)\left(B_{n}(\zeta)-B_{n}(z)\right)}{B_{n}(\zeta)(\zeta-z)} d \zeta, \quad z \in \operatorname{Int}(\gamma),
$$

where $\gamma$ is as in (21). Therefore, by (21) and (22), we obtain, for $z \in \operatorname{Int}(\gamma)$,

$$
R_{n}(f ; z)-r_{n}(f ; z)=\frac{1}{2 \pi} \int_{\gamma} \frac{f(\zeta)\left(B_{n}(\zeta)-B_{n}(z)\right)}{(\zeta-z)} \frac{d \zeta}{\left(B_{n}(\zeta)-1\right) B_{n}(\zeta)}
$$

Assume $1<\rho_{1}<\rho_{2}<\rho$. Let $\gamma(r)=\{z: \psi(z)=r\}, r>1$. (For the properties of $\gamma(r)$, see the remarks after the statement of Theorem 5 in Section 3.) Then, for $z \in \operatorname{Int}\left(\gamma\left(\rho_{1}\right)\right)$ and $\varepsilon \in(0, \rho-1)$, when $n$ is large enough, we have

$$
\left|R_{n}(f ; z)-r_{n}(f ; z)\right| \leq \frac{1}{2 \pi} \frac{\max _{\zeta \in \gamma\left(\rho_{2}\right)}|f(\zeta)|\left(\left(\rho_{2}+\varepsilon\right)^{n}+\left(\rho_{1}+\varepsilon\right)^{n}\right) l\left(\gamma\left(\rho_{2}\right)\right)}{\operatorname{dist}\left(\gamma\left(\rho_{1}\right), \gamma\left(\rho_{2}\right)\right)\left(\left(\rho_{2}-\varepsilon\right)^{n}-1\right)\left(\rho_{2}-\varepsilon\right)^{n}}
$$

where $l\left(\gamma\left(\rho_{2}\right)\right)$ denotes the length of $\gamma\left(\rho_{2}\right)$ and $\operatorname{dist}\left(\gamma\left(\rho_{1}\right), \gamma\left(\rho_{2}\right)\right)$ is the distance between $\gamma\left(\rho_{1}\right)$ and $\gamma\left(\rho_{2}\right)$. It follows that

$$
\limsup _{n \rightarrow \infty} \max _{z \in \gamma\left(\rho_{1}\right)}\left|R_{n}(f ; z)-r_{n}(f ; z)\right|^{1 / n} \leq \frac{1}{\rho_{2}} .
$$

Letting $\rho_{2} \nearrow \rho$ first and then letting $\rho_{1} \nearrow \rho$ yield

$$
\limsup _{n \rightarrow \infty} \max _{z \in \gamma(\rho)}\left|R_{n}(f ; z)-r_{n}(f ; z)\right|^{1 / n} \leq \frac{1}{\rho} .
$$

Now, from the fact that

$$
\frac{R_{n}(f ; z)-r_{n}(f ; z)}{B_{n}(z)}
$$

is analytic in $\mathbf{C} \backslash \mathbf{D}$ (including $\infty$ ), by using the maximum modulus principle, we have

$$
\max _{z \in \gamma\left(\rho_{2}\right)}\left|\frac{R_{n}(f ; z)-r_{n}(f ; z)}{B_{n}(z)}\right| \leq \max _{z \in \gamma\left(\rho_{1}\right)}\left|\frac{R_{n}(f ; z)-r_{n}(f ; z)}{B_{n}(z)}\right| .
$$

Thus, for $1<\rho_{1}<\rho_{2}$,

$$
\begin{aligned}
& \limsup _{n \rightarrow \infty} \max _{z \in \gamma\left(\rho_{2}\right)}\left|\frac{R_{n}(f ; z)-r_{n}(f ; z)}{B_{n}(z)}\right|^{1 / n} \\
& \quad=\limsup _{n \rightarrow \infty} \max _{z \in \gamma\left(\rho_{2}\right)} \frac{\left|R_{n}(f ; z)-r_{n}(f ; z)\right|^{1 / n}}{\rho_{2}} \\
& \quad \leq \max _{z \in \gamma\left(\rho_{1}\right)}\left|\frac{R_{n}(f ; z)-r_{n}(f ; z)}{B_{n}(z)}\right|^{1 / n} \\
& \quad=\limsup _{n \rightarrow \infty} \max _{z \in \gamma\left(\rho_{1}\right)} \frac{\left|R_{n}(f ; z)-r_{n}(f ; z)\right|^{1 / n}}{\rho_{1}}
\end{aligned}
$$


and so,

$$
\begin{aligned}
& \limsup _{n \rightarrow \infty} \max _{z \in \gamma\left(\rho_{2}\right)}\left|R_{n}(f ; z)-r_{n}(f ; z)\right|^{1 / n} \\
& \quad \leq \frac{\rho_{2}}{\rho_{1}} \limsup _{n \rightarrow \infty} \max _{z \in \gamma\left(\rho_{1}\right)}\left|R_{n}(f ; z)-r_{n}(f ; z)\right|^{1 / n} .
\end{aligned}
$$

This and (23) imply that, for $1<\rho_{*}<\rho$,

$$
\begin{aligned}
& \underset{n \rightarrow \infty}{\limsup } \max _{z \in \gamma\left(\rho_{*}^{2}\right)}\left|R_{n}(f ; z)-r_{n}(f ; z)\right|^{1 / n} \\
& \quad \leq \rho_{*} \limsup _{n \rightarrow \infty} \max _{z \in \gamma\left(\rho_{*}\right)}\left|R_{n}(f ; z)-r_{n}(f ; z)\right|^{1 / n} \\
& \quad \leq \rho_{*} \limsup _{n \rightarrow \infty} \max _{z \in \gamma(\rho)}\left|R_{n}(f ; z)-r_{n}(f ; z)\right|^{1 / n} \\
& \quad \leq \frac{\rho_{*}}{\rho}<1,
\end{aligned}
$$

which implies that $R_{n}(f ; z)-r_{n}(f ; z)$ converges to the limit zero locally and uniformly in $\operatorname{Int}\left(\gamma\left(\rho^{2}\right)\right)$ at a geometric rate. This finishes the proof of Theorem 5 .

\section{References}

1. I. N. Akhiezer, Theory of Approximation, Frederick Ungar Publ. Co., New York, 1956.

2. A. Bultheel, P. González-Vera, E. Henderiksen, and O. Njastad, Orthogonal Rational Functions, Cambridge Press, 1999.

3. _ Quadrature formulas on the unit circle based on rational functions, J. Comput. Appl. Math. 50 (1994), 159-170.

4. R. Brück, Extensions of Walsh's equiconvergence theorem for Jordan domains with analytic boundary curve, Acta Math. Hungar. 76 (1997), 155-171.

5. M. G. de Bruin and A. Sharma, Equiconvergence of some simultaneous Hermite-Padé interpolants: multiple nodes. In: Approximation Theory VIII, vol. 1, pp. 103-110, World Sci. Publishing, River Edge, NJ, 1995.

6. A. S. Cavaretta, Jr., H. P. Dikshit, and A. Sharma, Convergence of certain polynomial interpolants to functions defined on the unit circle, Acta Math. Hung. 53 (1989), 143-147.

7.

8. M. M. Djrbashian, A survey on the theory of orthogonal systems and some open problems. In: Orthogonal Polynomials: Theory and Practise (P. Nevai, ed.), pp. 135-146, Kluwer Academic Publisher, Dordrecht, 1990.

9. L. Fejér, Interpolation und konforme Abbildung, Göttinger Nachrichten (1918), 319-331.

10. D. Gaier, Lectures on Complex Approximation, Birkhäuser, Boston, 1987.

11. L. Kalmár, Über Interpolation, Mat. Lapok (1926), 120-149.

12. $\mathrm{X} . \mathrm{Li}$, Remarks on orthogonal polynomials with respect to varying measures and related problems, J. Math. Math. Sci. 2 (1993), 255-258.

13. _ Ranges of polynomials with curved majorants, Acta Math. Hungar. 65 (1994), 297-304.

14. X. Li, R. N. Mohapatra, and R. S. Rodriguez, Bernstein-type inequalities for rational functions with prescribed poles, J. London Math. Soc. 51 (1995), 523-531.

15. S. M. Lozinski, Über interpolation, Mat. Sbornik (N.S.) 8 (1940), 57-68.

16. F. Malmquist, Sur la détermination d'une classe de fonctions analytiques par luers valeurs dans un ensemble donné de points, C.R. Sixiéme Congrés (1925) Math. scandinaves (Kopenhagen) (1926), 253-259.

17. T. E. Price, Jr., Extensions of a theorem of J. L. Walsh, J. Approx. Theory 43 (1985), 140-150.

18. T. J. Rivlin, On Walsh equiconvergence, J. Approx. Theory 36 (1982), 334-345.

19. C. Runge, Theorie und Praxiz der Reihen, 1904, p. 137.

20. E. B. Saff and A. Sharma, On equiconvergence of certain sequences of rationa functions, Lecture Notes in Mathematics 1105, Springer-Verlag, New York, 1984, pp. 256-271.

21. E. B. Saff and J. L. Walsh, On the convergence of rational functions which interpolate in the roots of unity, Pacific J. Math. 45 (1973), 639-641. 
22. A. Sharma and P. Vertesi, Mean convergence and interpolation in roots of unity, SIAM J. Math. Anal. 14 (1983), 800-806.

23. S. Takenaka, On the orthogonal functions and a new formula of interpolation, Japan J. Math. 2 (1925), 129-145.

24. J. L. Walsh, On polynomial interpolation to an analytic function with singularities, Bull. Amer. Math. Soc. 38 (1932), 289-294.

25. _ Interpolation and Approximation (5th Edition), AMS Colloquium Publications, Vol. XX, Amer. Math. Soc., Providence, Rhode Island, 1969.

26. J. L. Walsh and A. Sharma, Least square approximation and interpolation in roots of unity, Pacific J. Math. 14 (1964), 727-730.

Department of Mathematics, University of Central Florida, Orlando, FL 32816 